\title{
Psychosocial work characteristics, need for recovery and musculoskeletal problems predict psychological distress in a sample of British workers
}

\author{
J.J DEVEREUX ${ }^{1}$, L.W. RYDSTEDT ${ }^{2}$ and M CROPLEY ${ }^{3 *}$ \\ ${ }^{1}$ Business Psychology Unit, University College London, UK \\ ${ }^{2}$ Lillehammer University College (HiL), AHS, Norway \\ ${ }^{3}$ Department of Psychology, University of Surrey, UK
}

*Corresponding author: Dr. Mark Cropley, Faculty of Arts and Human Sciences

University of Surrey, Guildford, Surrey, UK, GU2 7XH, email:mark.cropley@surrey.ac.uk 
From an original sample of 2454 participants free of self-reported psychological distress, 1463 workers completed a 15-month follow-up. Baseline measures included exposure to job demands, decision latitude, social support and need for recovery. Psychological distress was assessed using the General Health Questionnaire at baseline and at follow up. The findings showed that medium and high exposure to job demands and social support increased the risk of reporting psychological distress at 15 -months $(\mathrm{RR}=1.65 \&$ 1.45). The highest adjusted relative risk was observed for workers reporting a high need for recovery after work (RR 2.12, 1.90), and this finding was independent of the effects of job demands, decision latitude and social support. Neither decision latitude, nor low back problems increased the risk of reporting future psychological distress, although neck problems $(\mathrm{RR}=1.66)$ and hand/wrist problems $(\mathrm{RR}=1.45)$ did. It was concluded that need for recovery appears to be an important indicator of individual workers who are at risk of developing psychological distress long-term.

Keywords: Demands, social support, need for recovery, psychological distress

STATEMENT OF RELEVANCE This paper reports the findings of a longitudinal study showing that need for recovery from work was the strongest predictor, relative to psychosocial work characteristics (job demands, decision latitude, and social support), and musculoskeletal problems, of psychological distress 15 months later in individuals initially free from distress. 


\section{Introduction}

The experience of psychological distress in the general and work population can have serious health consequences (Dobson et al. 2009). As well as being an independent risk factor for cardiovascular disease (Stansfeld et al. 2002, van Amelsvoort et al. 2003), it is also very costly with respect to work absenteeism ( Hemingway el al. 1997, Stansfeld et al. 1997, Borritz et al. 2010, Jensen et al. 2010). Over the previous 30 years, high work demands, low decision latitude, and low levels of social support at work, have been identified as important constructs in relation to work related psychological well-being (Karasek, 1979; Johnson and Hall, 1988, van der Doef et al. 1999, de Lange et al. 2003, Hausser et al. 2010).

However longitudinal studies that have examined the impact of these work characteristics on the risk of psychological distress are limited in number. In an occupational cohort of middle-aged civil servants in London (Whitehall II cohort), for example, high job demands and low social support at work were the most important risk factors of future psychological distress (Stansfeld et al. 1997, Stansfeld et al. 1999). In a French study (Gazel), high job demands and low social support at work — based on the same measures developed by Johnson and Hall (1988) and Karasek et al., (1990) — were also among the most significant psychosocial predictors of subsequent depressive symptoms in both male and female employees working in a wide variety of occupations in a large national company in France (Niedhammer et al. 1998). Results from both the Whitehall II and Gazel Cohorts indicated that low decision latitude was a weaker predictor of psychological distress and depression.

One significant mechanism that is thought to underlie the relationship between work related demands (job demands and social support) and ill health is inadequate physical and psychological recovery from work demands. A high need for recovery after work can 
be regarded as a short-term adverse effect of high work related demands (Sluiter et al. 2003), and it is plausible that the need for recovery could be an intermediate factor between stressful work characteristics and psychological distress.

Simply put, work recovery may be thought of as the opposite of being exposed to the stressors at work. Efforts during work, mental or physical, lead to psychological or physiological load reactions, and these can be experienced as fatigue, stress or physiological arousal. During a work break, or once work has been completed, and the individual is no longer exposed to the demands of work, load reactions reverse, thus allowing recovery to occur (Cropley \& Zijlstra, in press; Meijman \& Mulder, 1998; Sluiter et al. 2001).

However, continuous exposure to high work demands and other psychosocial work factors may have long term health consequences. A recent 4-year follow-up study showed that high cortisol levels indicating physiological stress in office workers may be a likely reaction to long term job strain (high job demands, low decision latitude and low social support at work) (Rydstedt et al. 2008). Cortisol is a primary pathophysiological mechanism through which chronic stressors may be moderated by individual characteristics like need for recovery, which may increase the risk of disease long term (Rydstedt et al. 2009).

In a longitudinal study of 12,140 workers in the Maastricht cohort study, the need for recovery was found to be a strong predictor of subsequent cardiovascular disease and might be an intermediate factor between stressful work characteristics and cardiovascular disease (van Amelsvoort et al. 2003). The importance for the need for recovery as a potential intermediate factor between potentially stressful work characteristics and psychological distress has not been researched using a longitudinal study design. 
The relationship between musculoskeletal disorders, psychological distress and psychosocial risk have been investigated in several studies (Devereux et al. 1999, Davis and Heaney, 2000, Hoogendoorn et al. 2000, Kerr et al. 2001, Linton et al. 2001, Torp et al. 2001, Hoogendoorn et al. 2002, Devereux et al. 2004, Kjellberg and Wadman, 2007, Shaw et al. 2007, Gatchel et al. 2008, Keeley et al. 2008, Harrington and Feuerstein, 2010). Most studies have considered psychological distress as a predictor of musculoskeletal disorders. However, it is plausible that the pain, functional impairment or disability associated with musculoskeletal disorders may result in psychological distress. Thus theoretically, the relationship between musculoskeletal disorders and psychological distress may be bi-directional.

Musculoskeletal disorders and stress-related disorders are the leading causes of sickness absence in the UK. A relationship between these two disorders could potentially influence general medical and occupational health practice for managing psychological distress.

The present paper reports the findings from a prospective study that investigated whether psychosocial work characteristics, need for recovery and musculoskeletal problems predicted future psychological distress in a British working population.

\section{Methods}

\subsection{Study population}

Male and female workers within the age range 18-69 years were included in the study. Part-time workers and workers principally based long term within client organisations were excluded from the study sample. In all 3139 persons from 20 different companies within 11 industrial sectors responded to a baseline questionnaire. 
The prospective study comprised workers who did not report psychological distress at baseline $(\mathrm{N}=2454)$. The majority of the cohort, $63 \%$, were males, whereas $37 \%$ were females. The mean age was 42 years (S.D. 10.4 years). Late or night shift workers comprised $27 \%$ of the study sample. All nine major groups of the according to the UK Government Standard Occupational Classification were represented in the sample (http://www.ons.gov.uk). At the 15 month follow-up, 1724 subjects responded. Nonresponses to the follow-up questionnaire totalled 730 subjects.

\subsection{Psychosocial work characteristics}

The baseline questions used for measuring exposure to job demands, decision latitude, and social support have been used in a prospective study on British civil servants and possess good psychometric properties (Karasek et al. 1998, Marmot et al. 1991). The internal consistency (Cronbach alpha) values were: job demands $(4$ items $)=0.66$; Decision latitude $(15$ items $)=0.88$; and social support $=(7$ items $)=0.80$. Workers on a late or night shift were defined as having shift work.

\subsection{Need for recovery}

The need for recovery scale measured at baseline consisted of 11 questions containing a yes/no response on issues representing short term effects of a typical day of work such as "at the end of the working day I am really feeling worn out" and "after the working day I am often too tired to start other activities". Items are summed (1 item reversed) to give a total score between $0-11$. This scale has been evaluated against neuroendocrine activity and subjective health complaints (Sluiter et al. 2001). The internal consistency (Cronbach alpha) value was 0.83 . 


\subsection{Demographic and health related factors}

Age and gender were queried at baseline. Psychological distress was assessed using the General Health Questionnaire (GHQ-12) at baseline and at follow up. This scale consists of 12 items and it has been used in previous large scale studies to assess psychological distress (Rai et al. in press, Stansfeld et al. in press). The traditional scoring method $(0,0,1,1)$ was used to classify probable cases of psychological distress (scoring 3 or more points out of the 12 in total) (Goldberg, and Williams 1988; Goldberg et al., 1997, 1998). The internal consistency (Cronbach alpha) was 0.86 . 
Musculoskeletal problems experienced more than three times or lasting more than one week in the previous year classified individuals in the cohort as probable cases of lower back, neck or hand/wrist musculoskeletal disorders at baseline.

Using a 5\% sub-set of the follow-up population $(n=82)$, it was shown that subjects reporting problems in the lower back, neck and hands/wrists (between 26-30\% of subjects) were over 5 times more likely to also report difficulty performing everyday tasks compared to subjects without complaints when assessed against functional scales including the Revised Oswestry questionnaire, the neck disability index and the Disabilities of the Arm, Shoulder and Hand questionnaire (DASH). Comparing selfreports with a various range of motion, orthopaedic and palpation examination tests also showed that sensitivity measures varied between $0.37-0.63$ and specificity tests varied between 0.74-1.00 (Devereux et al. 2004). Previous research has shown that the questions used for assessing complaints have also demonstrated good to excellent testretest reliability and are recommended for use in epidemiological studies (Franzblau et al. 1997).

\subsection{Statistics}

Cox regression procedure, with a constant risk-period for all subjects, was used in all the analyses. Each work characteristic and need for recovery was divided into tertiles because of the non-normal distributions. The relationship between work characteristics and the cumulative incidence of psychological distress was first analysed. A crude relative risk and $95 \%$ confidence interval was determined for each stratum of job demands, decision latitude and social support at work. Low exposure to each of these variables was used as the internal reference group ( $R R=1)$. Age (less than 40 and 40 years or greater), gender and shift work were then included in a model with a single work 
characteristic to adjust for their potential confounding effects. A full model was then constructed using each work characteristic and adjusting for all confounders. The maximum correlation between independent variables was 0.25 (job demands and social support). Analyses were then performed in the same way to test the main effects of need for recovery and each musculoskeletal problem on the cumulative incidence of psychological distress.

Finally, analyses were conducted to examine to what extent the relationship between work characteristics and psychological distress was influenced by the need for recovery and musculoskeletal problems affecting the lower back, neck, and hands/wrists. For this purpose, multivariate analyses were performed on a model including all work characteristics with adjustment for age, gender and shift work, and either additional adjustment for subjective need for recovery, additional adjustment for low back problems, additional adjustment for neck problems, or additional adjustment for hands/wrist problems.

\section{Results}

In the population of workers who were not cases of psychological distress at baseline and with complete data on follow-up measurements $(n=1463)$, the cumulative incidence of psychological distress over the follow-up period was $26 \%(\mathrm{n}=381)$. Mean baseline GHQ scores, need for recovery and social support did not differ significantly between those who completed the follow-up and those 750 who did not respond. However job demands $(\mathrm{t}=2.52, \mathrm{p}<0.05)$ and decision latitude $(\mathrm{t}=3.55, \mathrm{p}<0.01)$ were significantly higher at baseline in those who completed the study relative to those who did not respond. 
As can be seen in Table 1, medium and high exposure to job demands increased the risk of reporting psychological distress at the 15 month follow-up (RR 1.31 to 1.56 ). The risk was greatest in the group with high job demands. There was little change in the relative risk after adjusting for age, gender and shift work. However, the relative risk for the high job demands group reduced from 1.62 to 1.56 after adjustment for job control and social support. The confidence intervals for the medium and high job demands after full adjustment indicated a true effect.

Medium and high exposure to low social support increased the relative risk by the same order of magnitude (RR 1.45). There was little change in the relative risk after adjusting for age, gender and shift work. Adjustment for job demands, decision latitude and potential confounders reduced the relative risk in the high exposure group from 1.47 to 1.38. The confidence intervals for the medium and high exposure groups after full adjustment indicated a true effect. Medium or high levels of exposure to poor decision latitude did not increase the risk of reporting future psychological distress.

Workers with a medium and high need for recovery after work were at an increased risk of reporting psychological distress at 15 months. Considering all the variables shown in Table 1, the highest adjusted relative risk (RR 2.12 and 1.90) was observed for workers reporting a high need for recovery after work. The increased risk was independent of the effects of job demands, decision latitude and social support variables. The confidence intervals for the medium and high groups after full adjustment indicated a true effect.

INSERT TABLE 1 AND 2 ABOUT HERE 
Low back problems did not increase the risk of reporting future psychological distress. However, a different relationship was observed for neck and hand/wrist problems. Neck problems and hand/wrist problems increased the risk of reporting future psychological distress. The relative risk was greatest for neck problems. The confidence intervals for these variables indicated a true effect after adjustment for age, gender, shift work and psychosocial work factors.

Table 2 shows the results of the multivariate analyses for psychological distress considering psychosocial work factors with different types of adjustment including need for recovery and low back, neck, hand/wrist problems. After allowing for the effects of need for recovery, a reduction in the relative risk for medium and high exposure to job demands was observed. The effect of exposure to high job demands was reduced from 1.56 to 1.32 (a reduction of $15 \%$ ) and the reduced effect size remained statistically significant. The effect of medium job demands was reduced by $11 \%$ but the reduced effect size was not statistically significant. Reductions in the relative risk for decision latitude and social support were also observed but the reductions were small in comparison to the reductions observed for job demands (up to 7\%).

After allowing for the effects of low back problems, a reduction in the relative risk for each psychosocial work factor was not observed (less than $0.5 \%$ ). After adjustment for neck problems, the largest reduction in the relative risk was observed for high job demands but the reduction was only $3 \%$. Similarly, allowing for the effects of hand/wrist problems had little influence over the relative risk for each psychosocial work factor. 


\section{Discussion}

The findings identified risk factors that predicted the onset of psychological distress at 15 months. High job demands and low social support at work were identified as significant risk factors. Even medium levels of exposure to these risk factors significantly increased the risk by $31-38 \%$. High job demands includes working very fast and intensively, not having enough time to do everything and having difficulty combining tasks. Low social support includes poor help, support and willingness to listen to workrelated problems from work colleagues and immediate superior and also poor quality of information. Poor decision latitude was not a significant risk factor. Poor decision latitude includes low control over work speed, breaks, decisions, environment and work, qualitative demands, ability to learn new things and task variation.

High job demands and low social support at work were also shown to predict psychological distress (assessed using the 30 item GHQ scale) in the Whitehall II study in England (Stansfeld et al. 1997). The relative risk of high job demands, after adjustment for age, employment grade and work characteristics, in the cohort of civil servants was 1.77 (95\% CI 1.5-2.2), similar to the relative risk in our study (RR 1.56 95\%CI 1.20-2.01), after similar adjustment. Likewise, relative risks for medium job demands were at the 1.30 level in both studies. For low social support at work, a higher relative risk was observed in the civil servant cohort (RR 1.61) compared to ours (RR $1.38)$.

In the present study, low decision latitude did not increase the risk of psychological distress. Similarly, in the Whitehall II study, low decision authority did not predict the onset of psychological distress (Stansfeld et al. 1997). Similar findings were also observed in the Maastricht cohort study comprising workers from 45 different Dutch companies. Low decision latitude was not a predictive risk factor, whereas job demands 
and social support at work were identified as risk factors (Bultmann et al. 2002). The same scales for measuring work characteristics and psychological distress were used in all three studies and the same findings were obtained in working populations. Furthermore, high psychological demands and low levels of social support at work were also shown to be significant predictors of subsequent depressive symptoms in the Gazel cohort study in France (Niedhammer et al. 1998).

Therefore, there is evidence from three high quality prospective studies to support our study findings that high job demands and low social support at work are risk factors for psychological distress, and low decision latitude does not increase risk. However, skill discretion, a form of job control like decision latitude, may be a separate predictor of psychological distress. Low decision latitude, measured by skill discretion and control over workload, was shown to predict depression in the Gazel study cohort (Niedhammer et al. 1998). In the Whitehall II study, low skill discretion, which includes having to repeat the same work, task variety, boredom, learning new things, skill level and initiative taking, did also increase the risk of psychological distress (Stansfeld et al. 1997). Future studies should include decision latitude and skill discretion as separate potential risk factors.

There may be an alternative explanation as to why decision latitude may not be an important risk factor in this study. There may be a difference in the relative importance of psychosocial work factors for different occupational groups. Rydstedt et al. (2007) showed that long term psychological distress was affected by high workload, high individual effort and insufficient rewards among managers/professionals but manual workers were more affected by time pressures, unwanted responsibilities and lack of social support. There may be occupational groups for which job control is more important. For example, Brasher et al. (2010) showed that autonomy/control was an 
important predictor of psychological distress among Royal Navy ship based, shore based and overseas personnel but not among Royal Navy sub-mariners.

It is important to note that need for recovery was also a risk factor for the onset of psychological distress. Furthermore, the relative risk for a high need of recovery was greater than the effect size for exposure to high job demands and low social support at work. This study offers a new finding that need for recovery from work is a predictor of psychological distress and should be further investigated using future longitudinal study designs in other populations and to examine whether it predicts physiological reactivity indicating a stress response. This new study finding is important because if the strength of this relationship is accurate, then need for recovery could be an important indicator to identify individual workers at risk of psychological distress.

Longitudinal studies that have measured need for recovery have shown that it is a predictor of subjective health complaints (in terms of psychosomatic complaints, emotional exhaustion, or sleep problems) and duration of future sickness absence (Sluiter et al. 2003). Therefore, it is plausible that need for recovery from work may predict psychological distress independent of exposure to job demands, decision latitude and social support at work and the effects of age, gender and shift work.

Results from the Whitehall II study suggested that the effects of working conditions on future mental health are likely to be mediated through individual perceptions of work (Stansfeld et al. 1999). The present study supports this view. The need for recovery from work can be viewed as an individual perception of the time required to recover from adverse working conditions that result in insufficient unwinding after exposure to stressful work characteristics (Jansen et al. 2002). Need for recovery is not necessarily an indication of physical exhaustion according to Bridger et al. (2010). Their study showed that need for recovery reflected a stressful or discordant work environment among 
seafarers where a level of frustration and annoyance was experienced on an average day at work.

According to our findings, adjusting for the effects of need for recovery resulted in a significant decrease in the relative risk of high job demands. This indicated that need for recovery played an intermediate role in the relationship between job demands and psychological distress, i.e., high job demands can lead to a perception that recovery after work is needed. This can subsequently trigger psychological distress. Nevertheless, high job demands was still an important independent risk factor after allowing for the effect of need for recovery. Therefore, the relationship between high job demands and psychological distress is partly independent from the effects of need for recovery.

There was no evidence to suggest that musculoskeletal problems played an intermediate role between psychosocial work factors and psychological distress. However, it was evident that neck and hand/wrist problems were independent risk factors for the onset of psychological distress. Such a relationship was not observed for low back problems. Musculoskeletal disorders affecting the neck and hand/wrists may impact the performance of everyday tasks more negatively than low back disorders in this cohort, thus, creating greater psychological distress.

The data collected using functional activity questionnaires on the lower back, neck and hands/wrists on a sub-sample of subjects supports this (Devereux et al. 2004). Furthermore, approximately one quarter of the study population were performing physical work involving manual handling/driving. About three quarters performed work involving static neck postures and repetitive hand movements (e.g. computer workers or production line workers). It has been shown that office workers performing more than 6 hours working on a computer per day in combination with either low decision authority, skill discretion or supervisor support report higher levels of neck pain and disability 
(Johnston et al. 2010). Hence, the study population was more likely to experience neck and hand/wrist musculoskeletal disorder problems.

The prevalence of lower back problems in the current study (about 1 in 4 workers) was greater than the prevalence of neck and hand/wrist problems (about 1 in 5 workers) at baseline in the cohort, as might be expected, but the nature of the work mainly performed by the cohort was likely to increase the risk of neck and hand/wrist problems by the greatest amount.

Some prospective studies have considered psychological distress as a predictor of musculoskeletal disorders (Feyer et al. 2000, Macfarlane et al. 2000, Devereux et al. 2004). A prospective study showed a non-significant relationship between psychological distress (GHQ12) and forearm pain experienced during the previous month and lasting more than a day after adjustment for repetitive arm movements, satisfaction with social support at work and illness behaviour score (Macfarlane et al. 2000). Likewise, a nonsignificant relationship was observed between psychological distress (GHQ12) and hand/wrist problems, as defined in this paper, after adjustment for age, gender, physical and psychosocial risk factors (Devereux et al. 2004).

Furthermore, a non-significant relationship was also observed for neck and low back problems. Only one prospective study has shown a significant predictive relationship between psychological distress (GHQ28) and new and recurrent episodes of low back pain among trainee nurses (Feyer et al. 2000). It is unclear whether psychological distress is a predictor of musculoskeletal disorders as too few prospective studies have been conducted. This study supports the hypothesis that musculoskeletal disorders predict the onset of psychological distress. However, it is acknowledged that a reciprocal relationship may also be possible. 
One possible limitation to the study was that our findings relied on self-reported data for all variables. Nonetheless, the scales used in this study have been previously validated against more objective methods and are reported to be suitable for use in epidemiological studies (Karasek et al. 1998, Sluiter et al. 2001, Stansfeld et al. 1992, Devereux et al. 2004).

Although the follow-up response rate was quite high, 730 workers failed to respond to the follow-up questionnaire (approximately 30\% of the cohort). Some workers who did not respond to the follow-up questionnaire may have done so because of leaving their employment, were absent from work at the time of the follow-up perhaps due to psychological distress or other reasons. It was not possible to determine the reasons for non-response. However, both job demands and decision latitude were significantly higher at baseline in those who completed the follow-up study relative to those who did not respond. This may imply that non-respondents may have had less concern and interest in the study because of their relatively lower job demands. It is unclear how the possibility of bias due to selective loss of subjects at follow-up would have affected the ability to detect a true effect between potential risk factors and psychological distress. In addition, 261 subjects ( $15 \%$ of the follow-up cohort) did not provide all necessary data. However, exclusion of these subjects had little effect on the crude relative risk for each independent variable (maximum 5.2\% change). Therefore, it can be concluded that this potential bias had limited effect on the study findings.

This research has important implications for interventions aimed at reducing psychological distress. Interventions may need to include reduction in musculoskeletal disorder symptom severity and also need for recovery from work activities. Researchers have proposed interventions in the workplace as an appropriate strategy for reducing mental ill health (Cox et al. 2010). There are various approaches that can be taken. Work 
design, organisation and management practice interventions that reduce high job demands and increase social support at work could lead to positive improvements in mental health.

The Health and Safety Executive Stress Management Standards (www.hse.gov.uk/stress/standards) are designed to help employers identify and avoid the risks associated with workload demands, job control, support issues, relationships, work roles, and organisational change. However, avoidance through work design, organisation and management practice interventions at an organisational level is not always a practicable solution and risks remain in the workplace. This present findings suggest that in conjunction with the Stress Management Standards, these risks need to be assessed and reduced at the individual level in accordance with the principles of prevention. Measuring the need for recovery among individual workers as part of a regular risk assessment may be an important step forward in evaluating risk, devising suitable interventions at the individual level and monitoring/reviewing the subsequent reduction in risk.

Nielsen et al. (2010) state that improvement in intervention studies are needed, and argue that other factors that target risk factors for health and wellbeing should be measured. According to the findings presented in this paper, perhaps organisations need to develop ways to help individual workers recover from workplace stressors. One possible way to lower the need for recovery from work is to address work scheduling. Companies may need to start examining specific work scheduling patterns to suite a specific task for individual workers. It is possible to monitor performance over time during a task, and develop specific guidelines when workers need to take a break for optimal performance and recovery. This practice is often overlooked and work-rest patterns are often selected arbitrarily or are designed around production/machine 
parameters. Work scheduling patterns may need to differ for workers with a high need for recovery.

An alternative strategy may be to include the use of flexible working to allow workers to select when reduced hours are needed temporarily to overcome fatigue or acute musculoskeletal discomfort, a precursor to long term musculoskeletal pain (Hamberg-van Reenen et al. 2008). Flexible working has become a useful strategy among some employers in light of the economic recession and the need to perform more work with fewer human resources (Confederation of British Industry, 2009).

\section{Acknowledgements}

We thank the Health and Safety Executive, UK for funding this study and also the reviewers for providing valuable comments during the review process.

\section{References}

Borritz, M., Christensen, K. B., Bültmann, U., Rugulies, R., Lund, T., Andersen, I., Villadsen, E., Diderichsen, F., and Kristensen, T. S., 2010. Impact of Burnout and Psychosocial Work Characteristics on Future LongTerm Sickness Absence. Prospective Results of the Danish PUMA Study among Human Service Workers. Journal of Occupational \& Environmental Medicine, 52, 964-970.

Brasher, K.S., Dew, A.B., Kilminster, S.G., and Bridger, R.S., 2010. Occupational stress in submariners: the impact of isolated and confined work on psychological well-being. Ergonomics, 53(3), 305-313.

Bridger, R.S., Brasher, K.S., and Dew. A., 2010. Work demands and need for recovery from work in ageing seafarers. Ergonomics, 53(8), 1006-1015.

Bultmann, U., Kant, I.J., Van Den Brandt, P.A., and Kasl, S.V., 2002. Psychosocial work characteristics as risk factors for the onset of fatigue and psychological distress: prospective results from the Maastricht Cohort Study. Psychological Medicine, 32, 333-45. 
Confederation of British Industry (CBI) 2009. Employment trends 2009:work patterns in the recession. CBI.

Cox, T., Taris, T.W., and Nielsen, K., 2010. Work and Stress Special Issue:organizational interventions: issues and challenges, Work and Stress, 24, 217-219.

Cropley, M. and Zijlstra, F.R.H. (in press). Work and Rumination. In Langan-Fox, J \& Cooper, C.L. (Eds).

Handbook of Stress in the Occupations. U.K: Edward Elgar Publishing Ltd.

Davis, K.G., and Heaney, C.A., 2000, The relationship between psychosocial work characteristics and low back pain: underlying methodological issues. Clinical Biomechanics, 15, 389-406.

De Lange, A. H., Taris, T. W., Kompier, M A J., Houtman, I. L. D., and Bonger S, P. M. 2003. The very best of the millennium": Longitudinal research and the Demand-Control-(Support) model. Journal of Occupational Health Psychology, 8, 282-305.

Devereux, J., Rydstedt, L., Kelly, V., Weston, P., and Buckle, P., 2004, The role of work stress and psychological factors in the development of musculoskeletal disorders: The Stress and MSD Study. Sudbury: HSE Books.

Devereux, J.J., Buckle, P.W., and Vlachonikolis, I.G., 1999. Interactions between physical and psychosocial work risk factors increase the risk of back disorders: An epidemiological study. Journal of Occupational \& Environmental Medicine, 56, 343-53.

Dobson, M., and Schnall, P. L., 2009. From stress to distress: The impact of work on mental health. In: Unhealthy work: Causes, consequences, cures. Schnall, P. L., Dobson, M., Rosskam, E., Amityville, N.Y.. Baywood, 113-132.

Feyer, A.M., Herbison, P., Williamson, A.M., De Silva, I., Mandryk, J., Hendrie, L. et al., 2000, The role of physical and psychological factors in occupational low back pain: a prospective cohort study. Journal of Occupational \& Environmental Medicine, 57, 116-20.

Franzblau, A., Salerno, D.F., Armstrong, T.J., and Werner, R.A., 1997. Test-retest reliability of an upper extremity discomfort questionnaire in an industrial population. Scandinavian Journal of Work Environment and Health, 23, 299-307.

Gatchel, R. J., Bernstein, D., Stowell, A. W., and Pransky, G., 2008. Psychosocial differences between highrisk acute vs. chronic low back pain patients. Pain Practice, 8, 91-97.

Goldberg, D.P. and Williams, P. (1988). A user's guide to the General Health Questionnaire. Windsor: NFER-Nelson.

Goldberg, D.P., Gater, R., Sartorius, N., Ustun, T.B., Piccinelli, M., Gureje, O. and Rutter, C., 1997. The validity 
of two versions of the GHQ in the WHO study of mental illness in general healthcare. Psychological Medicine, 27, 191-197.

Goldberg, D.P., Oldehinkel, T. and Ormel, J., 1998. Why GHQ threshold varies from one place to another. Psychological Medicine, 28, 915-921.

Hamberg-Van Reenen, H.H., Van Der Beek, A.J., Blatter, B., Van Der Grinten, M.P., Van Mechelen, W., 2008. Does musculoskeletal discomfort at work predict future musculoskeletal pain? Ergonomics, 51(5), 637-648.

Harrington, C. B., Feuerstein, M., 2010. Work style in Office Workers: Ergonomic and Psychological Reactivity to Work Demands. Journal of Occupational \& Environmental Medicine, 52, 375-382.

Hausser, J.A., Mojzisch, A., Niesel, M., and Schulz-haedt, S., 2010, Ten years on: A review of a recent research on the Job Demand- Control (- Support) model and psychological well-being. Work and Stress, $24,1-35$.

Hemingway, H., Shipley, M., Stansfeld, S.A., and Marmot, M., 1997. Sickness absence from back pain, psychosocial work characteristics and employment grade among office workers. Scandinavian Journal of Work Environment and Health, 23, 121-9.

Hoogendoorn, W.E., Bongers, P.M., Vet, H.C.W.D., Houtman, I.L.D., Ariëns, G.A.M., and Mechelen, W.V. ET AL., 2002, Psychosocial work characteristics and psychological strain in relation to low back pain: results of a prospective cohort study. Scandinavian Journal of Work, Environment and Health, 27, 258-67.

Hoogendoorn, W.E., Van Poppel, M., Bongers, P.M., Koes, B.W., and Bouter, L.M., 2000. Systematic review of psychosocial factors at work and private life as risk factors for back pain. Spine, 25, 2114-25.

Jansen, N. W. H., Kant, I.J., and Van Den Brandt, P. A., 2002. Need for recovery in the working population: Description and associations with fatigue and psychological distress. International Journal of Behavioural Medicine, 9, 322-340.

Jensen, J. N., Karpatschof, B., Labriola, M., and Albertsen, K., 2010. Do Fear-Avoidance Beliefs Play a Role on the Association Between Low Back Pain and Sickness Absence? A Prospective Cohort Study Among Female Health Care Workers. Journal of Occupational \& Environmental Medicine, 52, 85-90.

Johnson, J.V., and Hall, E.M., 1988. Job strain, work place social support, and cardiovascular disease: A cross- sectional study of a random sample of the Swedish working population. American Journal of Public Health, 78, $1336-1342$. 
Johnston, V., Jull, G., Souvlis, T., and Jimmieson, N.L., 2010. Interactive effects from self-reported physical and psychosocial factors in the workplace on neck pain and disability in female office workers. Ergonomics, 53(4), 502-513.

Karasek, R. A., 1979, Job demands, job decision latitude and mental strain: Implications for job redesign. Administrative Science Quarterly, 24, 285-308.

Karasek, R. A., Brisson, C., Kawakami, N., Houtman, I., Bongers, P., and Amick, B., 1998. The Job Content Questionnaire (JCQ): An instrument for internationally comparative assessments of psychosocial job factors. Journal of Occupational Health Psychology, 3, 322-55.

Keeley, P., Creed, F., Tomenson, B., Todd, C., Borglin, G., and Dickens, C., 2008. Psychosocial predictors of health-related quality of life and health service utilisation in people with chronic low back pain. Pain, 135, 142-150.

Kerr, M.S., Frank, J.W., Shannon, H.S., Norman, R.W., Wells, R.P., Neumann, W.P. et al., 2001. Biomechanical and psychological risk factors for low back pain at work. Journal of Public Health, 91, 1069-75.

Kjellberg, A., and Wadman, C., 2007. The role of the affective stress response as a mediator of the effect of psychosocial risk factors on musculoskeletal complaints-Part 1: Assembly workers .International Journal of Industrial Ergonomics, 37, 367-374.

Linton, S.J., 2001. Occupational psychological factors increase risk of back pain: a systematic review. Journal of Occupational Rehabilitation, 11, 53-66.

Macfarlane, G.J., Hunt, I.M., and Silman, A., 2000, Role of mechanical and psychosocial factors in the onset of forearm pain: prospective population based study. British Medical Journal, 321, 676-86.

Marmot, M., Smith, G.D., Stansfeld, S.A., PateL, C., North, F., Head, J., ET AL., 1991, Health inequalities among British civil servants: The Whitehall II Study. The Lancet, 337, 1387-93.

Meijman, T.F. \& Mulder, G. (1998). Psychological aspects of workload. In P.J.D. Drenth, H. Thierry, \& C.J. de Wolff (Eds.), Handbook of work and organizational psychology: Vol. 2 Work Psychology (pp.5-33). Hove: Psychology Press.

Niedhammer, I., Goldberg, M., Leclerc, A., Bugel, I., and David, S., 1998. Psychosocial factors at work and subsequent depressive symptoms in the Gazel Cohort. Scandinavian Journal of Work, Environment and Health, 24, 197-205. 
Nielsen, K., Taris, T.W., and Cox, T., 2010, The future of organizational interventions:addressing the challenges of today's organizations. Work and Stress Special Issue:organizational interventions: issues and challenges, Work and Stress, 24, 219-233.

Rai, D., Kosidou, K., Lundberg, M., Araya, R., Lewis, G., and Magnusson C. in press. Psychological distress and risk of long-term disability: population-based longitudinal study. Journal of Epidemiology and Community Health.

Rydstedt, L.W., Cropley, M., Devereux, J.J., and Michalianou, G., 2009. The Effects of Gender, Long-Term Need for Recovery and Trait Inhibition-Rumination on Morning and Evening Saliva Cortisol Secretion. Anxiety, Stress and Coping, 22(4), 465-474.

Rydstedt, L., Cropley,M., Devereux, J., and Michallianou, G., 2008. The Relationships between Long-term IsoStrain and Morning and Evening Saliva Cortisol Secretion among White-Collar Workers. Journal of Occupational Health Psychology, 13(2), 105-113.

Rydstedt, L., Devereux, J. and Sverke, M. 2007. Comparing and combining the demand-control-support model and the effort reward imbalance model to predict long-term mental strain. European Journal of Work and Organizational Psychology, 16(3), 261-278.

Shaw, W. S., Means-Christensen, A., Slater, M. A., Patterson, T. L., Webster, J. S., and Atkinson, J. H., 2007, Shared and independent associations of psychosocial factors on work status among men with sub acute low back pain. The Clinical Journal of Pain, 23, 409-416.

Sluiter, J.K., De Croon, E.M., Meijman, T.F., Frings-Dresen, M.H.W., 2003. Need for recovery from work related fatigue and its role in the development and prediction of subjective health complaints. Journal of Occupational \& Environmental Medicine, 60, 62-70.

Sluiter, J.K., Frings-Dresen, M.H.W., Van Der Beek, A.J., Meijman, T.F., 2001. The relation between workinduced neuroendocrine reactivity and recovery, subjective need for recovery, and health status. Journal of Psychosomatic Research, 50, 29-37.

Stansfeld SA, Fuhrer R, Head J. (in press). Impact of common mental disorders on sickness absence in an occupational cohort study. Occupational Environmental Medicine.

Stansfeld, S.A., Fuhrer, R., head, J., Ferrie, J., Shipley, M., 1997, Work and psychiatric disorder in the Whitehall II Study. Journal of Psychosomatic Research, 43, 73-81.

Stansfeld, S.A., Fuhrer, R., Shipley, M., and Marmot, M., 1999. Work characteristics predict psychiatric disorder: prospective results from the Whitehall II study. Journal of Occupational \& Environmental Medicine, 56, 302-7. 
Stansfeld, S.A., Fuhrer, R., Shipley, M., 2002. Psychological distress as a risk factor for coronary heart disease in the Whitehall II study. International Journal of Epidemiology, 31, 248-55.

Stansfeld, S.A., Marmot, M., 1992. Social class and minor psychiatric disorder in British Civil Servants: a validated screening survey using the General Health Questionnaire. Psychological Medicine, 22, 739-49.

Torp, S., Riise, T., and Moen, B.E., 2001. The impact of psychosocial work factors on musculoskeletal pain: a prospective study. Journal of Occupational \& Environmental Medicine, 43, 120-6.

Van Amelsvoort, L.G.P.M., Kant I.J., Bültmann, U., and Swaen, G.M.H., 2003. Need for recovery after work and the subsequent risk of cardiovascular disease in a working population. Journal of Occupational \& Environmental Medicine, 60, 83-87.

Van Der Doef, M., and Maes, S., 1999. The job demand-control (-support) model and psychological wellbeing: A review of 20 years of empirical research. Work and Stress, 13, 87-114. 
Table 1 Relative risks of independent variables on the cumulative incidence of psychological distress 


\begin{tabular}{|c|c|c|c|c|c|}
\hline $\begin{array}{l}\text { Factor and } \\
\text { exposure lev }\end{array}$ & $(\%)$ & $N$ & Crude RR (95\% CI) & Adjusted RR $^{\mathrm{a}}(\mathbf{9 5 \%} \mathrm{CI})$ & Adjusted RR $^{\mathrm{b}}(\mathbf{9 5 \%} \mathrm{CI})$ \\
\hline \multicolumn{6}{|l|}{ Job demands } \\
\hline - low & 20.3 & 595 & 1.00 & 1.00 & 1.00 \\
\hline - medium & 27.3 & 495 & $1.34(1.05-1.28)$ & $1.33(1.04-1.70)$ & $1.31(1.02-1.68)$ \\
\hline - high & 33.5 & 373 & $1.65(1.28-2.12)$ & $1.62(1.26-2.09)$ & $1.56(1.20-2.01)$ \\
\hline \multicolumn{6}{|c|}{ Decision latitude } \\
\hline - low & 26.8 & 570 & 1.00 & 1.00 & 1.00 \\
\hline - medium & 23.7 & 486 & $0.88(0.69-1.12)$ & $0.89(0.70-1.14)$ & $0.89(0.70-1.13)$ \\
\hline - high & 27.8 & 407 & $1.03(0.81-1.32)$ & $1.11(0.86-1.42)$ & $1.04(0.80-1.35)$ \\
\hline \multicolumn{6}{|c|}{ Social support } \\
\hline - low & 21.8 & 822 & 1.00 & 1.00 & 1.00 \\
\hline - medium & 31.6 & 190 & $1.45(1.08-1.94)$ & $1.48(1.11-1.98)$ & $1.43(1.06-1.92)$ \\
\hline - high & 31.5 & 451 & $1.45(1.16-1.80)$ & $1.47(1.18-1.84)$ & $1.38(1.09-1.74)$ \\
\hline \multicolumn{6}{|c|}{ Need for Recovery } \\
\hline - low & 16.9 & 561 & 1.00 & 1.00 & 1.00 \\
\hline - medium & 27.3 & 436 & $1.61(1.23-2.11)$ & $1.61(1.23-2.11)$ & $1.52(1.16-2.00)$ \\
\hline - high & 35.8 & 466 & $2.12(1.65-2.72)$ & $2.12(1.65-2.73)$ & $1.90(1.46-2.47)$ \\
\hline \multicolumn{6}{|c|}{ Low back problems } \\
\hline- no & 25.5 & 1080 & 1.00 & 1.00 & 1.00 \\
\hline - yes & 27.7 & 383 & $1.09(0.87-1.36)$ & $1.11(0.88-1.38)$ & $1.08(0.86-1.35)$ \\
\hline \multicolumn{6}{|c|}{ Neck problems } \\
\hline- no & 23.6 & 1230 & 1.00 & 1.00 & 1.00 \\
\hline - yes & 39.1 & 233 & $1.66(1.31-2.10)$ & $1.66(1.31-2.11)$ & $1.55(1.22-1.98)$ \\
\hline \multicolumn{6}{|c|}{ Hand/wrist problems } \\
\hline - no & 24.2 & 1221 & 1.00 & 1.00 & 1.00 \\
\hline - yes & 35.1 & 242 & $1.45(1.14-1.84)$ & $1.44(1.13-1.84)$ & $1.37(1.07-1.75)$ \\
\hline
\end{tabular}

$\mathrm{RR}$, relative risk; CI, confidence interval

${ }^{a}$ Adjusted for age, gender and shift work

${ }^{\mathrm{b}}$ Adjusted for age, gender, shift work, and other work characteristics 
Table 2. Relative risks of psychosocial work factors on the cumulative incidence of psychological distress after different types of adjustment 
Factor and Adjusted RR ${ }^{\mathrm{a}}$

exposure level

(95\% CI)
$\mathbf{R R}^{\mathrm{a}}(95 \% \mathrm{CI})$

$\begin{array}{llll}\text { Additional } & \text { Additional } & \text { Additional } & \text { Additional } \\ \text { adjustment for } & \text { adjustment for } & \text { adjustment for } & \text { adjustment for } \\ \text { need for } & \text { low back } & \text { neck problems } & \text { hand/wrist } \\ \text { recovery } & \text { problems } & & \text { problems }\end{array}$

Job demands

- low

$$
1.00
$$

1.00

1.00

1.00

1.00

- medium

1.31 1.02-1.68)

1.17 (0.91-1.50)

1.31 (1.02-1.67)

1.29(1.01-1.65)

1.31 (1.02-1.68)

- high

$1.56(1.20-2.01)$

1.32 (1.01-1.73)

1.51 (1.17-1.95)

$1.56(1.21-2.02)$

Decision latitude

- low

$\begin{array}{ll}1.00 & 1.00 \\ 0.89(0.70-1.13) & 0.86(0.67-1.10)\end{array}$

1.00

1.00

1.00

- medium

$\begin{array}{ll}0.89(0.70-1.13) & 0.86(0.67-1.10) \\ 1.04(0.80-1.35) & 0.97(0.75-1.26)\end{array}$

$0.89(0.70-1.13)$

$0.88(0.69-1.13)$

0.89 (1.13-1.33)

- high

$1.04(0.80-1.35)$

$1.02(0.79-1.33)$

$1.02(0.79-1.33)$

Social support

- low

1.00

1.00

1.00

1.00

1.00

- medium

$1.43(1.06-1.92) \quad 1.38(1.03-1.86)$

1.43 (1.07-1.92)

$1.40(1.04-1.88)$

$1.38(1.03-1.86)$

- high

$1.38(1.09-1.74) \quad 1.32(1.04-1.66)$

1.38(1.09-1.73)

$1.35(1.07-1.70)$

$1.35(1.07-1.70)$

$\mathrm{RR}$, relative risk; $\mathrm{CI}$, confidence interval

${ }^{a}$ Adjusted for age, gender, shift work, and other psychosocial work factors 